\title{
El estudio de las redes personales para la implementación de estrategias de atención sociomédica
}

\author{
Dra. Margarita Terán Trillo - Universidad Nacional Autónoma de México
}

\section{Resumen}

Este estudio comparativo entre enfermos geriátricos sin problema cognitivo y cuidadores de pacientes con demencia, detectó diferencias importantes en la integración social ( 2.72 y 1.71 respectivamente) y número de personas en su red ( 7.60 y 3.95); estableciendo las bases para generar estrategias de atención que incrementen sus redes e influir en una mayor calidad de vida.

Palabras clave: Red personal - enfermos - cuidadores - calidad de vida

\section{Abstract}

This comparative study between patients geriatric's without cognitive problem and Care taker of patient with dementia, detected important differences in the social integration ( 2.72 and 1.71 respectively) and number of people in his net ( 7.60 and 3.95); Establishing the bases to generate strategies of attention that increase his nets and to have an influence on main quality of life.

Key words: Personal network - patients- care taker - quality of life

\section{I ntroducción}

El estudio, análisis y representación de las redes constituye un importante complemento en el abordaje de distintas situaciones, necesidades o problemas del ámbito de la salud, ya que permite obtener una visión más amplia de los factores que se articulan de forma directa e indirecta en su desarrollo. También facilita la medición de estructuras sociales que emergen de las relaciones entre diversos sujetos o entidades sociales involucradas, considerando en todo caso, los aspectos normativos y de bioética. El punto de partida es la información de las relaciones sobre los cuales se pretende identificar los vínculos factibles de ser analizados en variables contextuales e indicadores, que suelen corresponder a un determinado nivel de atención, servicio o padecimiento específico.

Con fines operativos, se ha considerado aquí, que la red social es la trama de interrelaciones profesionales que se generan entre el equipo de salud y la población que recibe atención; enfocadas al establecimiento de relaciones significativas con el sujeto sano o enfermo y su la familia, en forma especial con

\footnotetext{
1 Profesora de tiempo completo en la Escuela Nacional de Trabajo Social-UNAM. Responsable del estudio de redes sociales y percepción de calidad de vida, del proyecto "Demencia senil y Alzheimer: detección temprana", perteneciente al Megaproyecto Nuevas Estrategias Epidemiológicas, Genómicas y Proteómicas en Salud Pública, registro SDEI.PTID.05.5, coordinado por la Facultad de Medicina, dentro del Programa Transdisciplinario en Investigación y Desarrollo para Facultades y Escuelas de la Universidad Nacional Autónoma de México. Correo electrónico: mteran@unam.mx
} 
REDES- Revista hispana para el análisis de redes sociales

Vol.17,\#8, Diciembre 2009

http://revista-redes.rediris.es

quienes hace la función de responsables o cuidadores. Esto constituye una primera red, una red mediadora, que tiende a conocer la red personal de aquellos que requieren de mayor apoyo, y se extiende a todos los elementos capaces de proporcionar algún recurso real o potencial a nivel personal, familiar, grupal e institucional, con la finalidad de afrontar los distintos costos (emocionales, sociales y económicos) que se plantean en este ámbito.

Cada red mantiene características propias que la determinan como una totalidad dinámica que regula el comportamiento social de las personas implicadas. "Las posiciones ocupadas por lo actores participantes de la red son puestas de manifiesto como factores explicativos cruciales de sus oportunidades, constricciones y márgenes de maniobra para la acción" (De Federico 2005:51); el conocimiento de sus propiedades permite comprender, predecir e incluso obtener mejores resultados cuando se trata de fortalecer el capital relacional (Molina 2005, Lin 2001), indispensable en la atención sociomédica. Actualmente existen proyectos promovidos por algún grupo vulnerado por una enfermedad en particular, que organizado, logra la convocatoria y participación de elementos de distintos sectores para la obtención de recursos curativos y paliativos, pero que trascienden a la atención preventiva por la difusión que alcanza. 2

Se trabaja principalmente con las redes personales de la población atendida y se requiere de identificarlas para generar intervenciones que incidan favorablemente en la prevención, mantenimiento o recuperación de la salud, enfocados a una mayor calidad de vida en los sujetos sanos (medicina familiar, general y preventiva) en el primer nivel de atención. También en la procuración de la atención medica de alta especialidad que corresponden al segundo y tercer nivel de servicios, e incluye los cuidados paliativos y apoyo tanatológico con pacientes en fase terminal (Hospitales, Institutos y Centros de alta especialidad).

La red personal o red egocéntrica alude al mundo social de cada sujeto; se integra a lo largo de la vida, por diversos los lazos afectivos y funcionales surgidos en relaciones cotidianas. Los lazos fuertes tienen una mayor consistencia o intensidad emocional, confianza mutua y reciprocidad (De Grande 2007, Maya 2002, Requena 1996, Granovetter 1973); estos lazos se profundizan cuando existe una alta de convivencia, como es la familia y los amigos. En tanto que los lazos funcionales

\footnotetext{
2 Se conocen muchas experiencias de este tipo, porque constituyen verdaderas campañas de sensibilización masiva como ha sucedido con el cáncer de mama o del VIH, que rebasan el ámbito de los recursos exclusivamente de las instituciones de salud, al establecer nexos con algunas empresas productoras de medicamentos, de enceres domésticos; con medios de comunicación, a través de videos, por internet, promoción televisiva, radio, carteles, trípticos, etcétera. Lo que demuestra el valor del trabajo basado en redes mixtas.
} 
REDES- Revista hispana para el análisis de redes sociales

Vol.17,\#8, Diciembre 2009

http: //revista-redes.rediris.es

guardan más relación con aspectos instrumentales, en donde se ubican vecinos compañeros de trabajo y algunos proveedores de servicios.

El mayor aporte de las redes personales en atención a la salud, se manifiesta en la identificación de los elementos que la integran, los diversos apoyos que portan, la manera en que articulan sus funciones; los mecanismos de vinculación a otras personas; las formas específicas de su gestión y activación; o bien, para conocer los factores o condiciones para la reactivación y reorientación de su dinámica, particularmente para reforzar el tratamiento médico a través de intervenciones sociales concretas.

Se conoce ampliamente la influencia que ejercen las redes personales en la retroalimentación sobre aspectos de cuidados a salud, rutina de ejercicio, dietas; pero sobre todo, en la asistencia oportuna al médico y apego a los tratamientos; el mejor afrontamiento en procesos crónicos y degenerativos, o ante pérdidas 0 crisis. 3

\section{Antecedentes}

Uno de nuestros primeros estudios de redes sociales con cuidadores data de 2002, en una Clínica de Medicina Familiar del ISSSTE", logrando identificar las condiciones y apoyos de 38 cuidadores principales. En donde un 84\% correspondió a mujeres y el $16 \%$ a hombres con un promedio 55 años de edad; $64 \%$ trabajan en el hogar, tenía estudios de nivel medio; esposa, madre, hija o hermana del enfermo dependiente por enfermedad crónico degenerativa y/o discapacidad; con una mediana de 10.5 en años de cuidados, una dedicación en horas diarias de 2024 un $26 \%$ y $24 \%$ de $10-14$ horas; que se auto consideraba cuidador por obligación el $50 \%$, por deber moral el $24 \%$ y por afecto el $21 \%$.

\footnotetext{
${ }^{3}$ Litman encontró la incidencia entre factores estresantes en la familia, sucedidos con frecuencia en un periodo de dos las semanas anteriores a la aparición de alguna infección por estreptococos en población infantil, concluyendo que las crisis familiares habían disminuido la resistencia orgánica a la infección (Litman 1982). La mayor o menor presencia de red personal en embarazadas marcó la diferencia de una mayor asistencia a consultas (Infante 1990). O en el caso de pacientes con trastornos de depresión, cuya red se constituye en fuente de recursos (Caraveo 1997), de igual manera ha sucedido en estudios de pacientes con diabetes mellitus (Rodríguez y Guerrero 1997, Rodríguez, Díaz y García 1995), hipertensión, problemas biliares o cardiopatías. Un estudio reciente con enfermos del corazón, ha establecido la correlación entre los cambios bioquímicos producidos por la enfermedad y los ocasionados por las interacciones sociales (Loucks y cols. 2006).

Desde el Hospital también se han realizado muy diversos estudios. Uno de ellos ha hecho la detección de las alteraciones ocurridas en la dinámica familiar derivadas de la muerte materna post-parto, entre mucha otra información, destaca el hecho de que los recién nacidos al cuidado de los abuelos alcanzaron un $85.1 \%$ al egresar del hospital y la sobrevivencia de un año en la casi totalidad, ya que sólo hubo una defunción (Reyes, Bobadilla, Karchmer y Martínez 1998). Y serían muchos los casos en que se demuestra el valor de la red en población atendida en servicios de salud, siendo el núcleo familiar el que proporciona mayor soporte.

${ }^{4}$ González-Ortiz María Angélica, Terán-Trillo Margarita, Ponce-Rosas Efrén Raúl y Sánchez-Escobar Laura Elena (ver cita completa en la bibliografía).
} 
REDES- Revista hispana para el análisis de redes sociales

Vol.17,\#8, Diciembre 2009

http: //revista-redes.rediris.es

El $74 \%$ de cuidadores presentó alguna enfermedad, 42\% cursaba estadios de insomnio; el $53 \%$ se sentía tranquilo, $42 \%$ manifestaba estrés y cansancio, angustia o depresión; el $5 \%$ en el límite de sus fuerzas, $66 \%$ requería de analgésicos solos o combinados.

El apoyo familiar representó un $72 \%$ del total (en 35\% se trato de descendentes, $28 \%$ de iguales y $9 \%$ de ascendentes) y sólo el $37 \%$ pedía ayuda extrafamiliar. El $79 \%$ de enfermos mantenía un buen trato al cuidador, en tanto que el $11 \%$ se manejaba con actitudes de maltrato. En cuanto a la red institucional, la totalidad de enfermos (en la mayoría de los casos también el cuidador), tenían acceso a los servicios del ISSSTE; adicionalmente, el 66\% acudía a otros servicios de salud, ya que $29 \%$ también tenía derecho al IMSS y $26 \%$ recurría a la Secretaria de Salud. Algunos de ello recurrían a servicios religiosos. La mayoría de los cuidadores deseaban recibir más orientación médica y social para: mejorar los cuidados al enfermo, mejorar sus relaciones familiares, disponer de atención psicosocial y apoyos económicos (González, Terán, Ponce y Sánchez 2003).

Esto resultados, aunados a las propuestas hechas por el grupo estudiado, han llevado a la revisión de algunos instrumentos ${ }^{5}$ que identifiquen elementos y el flujo de recursos en la red personal, ya que en nuestro caso, el principal objetivo esta en trabajar con las redes para optimizar recursos a través de intensificar las interrelaciones, generar corresponsabilidad y la auto-organización. Finalmente se optó por desarrollar un cuestionario propio operable en nuestro contexto; retomando los planteamientos de diversos autores, principalmente de De la Revilla (1991), Requena (1996) y Molina (2001).

\section{Objetivo}

Analizar las redes personales en fracciones de dos grupos (de los tres que integran la muestra total de la investigación sobre "Demencia senil y Alzheimer"), tendiente al desarrollo de estrategias de intervención para el fortalecimiento y ampliación de estas redes.

5 Bellón JA, Delgado A, Luna del CD y Lardelli CP. (1996) para la Escala de Duke UNC II. Díaz Veiga (1987), ver referencias. 
REDES- Revista hispana para el análisis de redes sociales

Vol.17,\#8, Diciembre 2009

http: //revista-redes.rediris.es

\section{Metodología}

Se trata de un estudio longitudinal, descriptivo y comparativo en sujetos provenientes de dos grupos:

Grupo de Pacientes Geriátricos (GPG), mayores de 60 años y más, sin problema cognitivo, atendidos en el servicio de geriatría de un Hospital de Seguridad Social (ISSSTE); por tratarse de un grupo de alto riesgo por edad.

Grupo de Cuidadores (GC) familiares de enfermos con demencia atendidos en una "Residencia de día" (servicio de Asistencia Privada). Aún cuando la mayoría están por debajo de los 60 años de edad, se han incluido aquí por el riesgo que implica un 5 a 10\% de factor genético con relación a demencias y Alzheimer; aunado a su desgaste por su condición de cuidador. También en la consideración de que su red personal se ha vuelto la red de apoyo indirecto del enfermo, ante la imposibilidad de este último para mantener su propia red.

Se utilizaron dos instrumentos de medición: El Cuestionario "Breve de Calidad de Vida, CUBRECAVI", mismo que integra 9 áreas: salud (subjetiva, objetiva y emocional), integración social, habilidades funcionales, actividad y ocio, calidad ambienta, satisfacción con la vida, educación, ingresos, servicios sociales y de salud (mismo que esta validado y estandarizado, (adaptado para población mexicana en los rubros de monto de ingresos). Y el cuestionario-inventario "Red Social" ${ }^{\prime 6}$ que consta de 5 áreas; probado, validado y estandarizado en instituciones de salud y asistenciales. La primera área corresponde a datos de identificación del ego (persona entrevistada), antecedida de una breve explicación del tema y recordatorio del consentimiento informado, ${ }^{7}$ reforzando lo expuesto al inicio de la entrevista, además de haber firmado previamente la "Carta de consentimiento informado y participación voluntaria". La segunda se refiere a un generador de nombre que identifica los elementos de la red (alteris), tipo de relación (lazos fuertes y débiles), el tipo de apoyo que proporcionan (atribución de capital) y hacia quién va dirigido principalmente el apoyo (direccionalidad). La tercera mide la participación en grupos de la institución y fuera de ella. La cuarta, el acceso a diversos servicios y la quinta, a la presencia de apoyos sustitutivos y simbólicos. Estas características facilitan observar estructura y dinámica de la red: los

\footnotetext{
6 Red Social. Terán -Trillo M. Registro: INDA-RPDA No. O3 200506141111270001

7 Se mantuvo la observancia para investigaciones en salud contenida en la Ley General de Salud en México; por lo que se apega a lo establecido en el título segundo, capítulo I, artículo 17, en donde se hace referencia a investigación sin riesgo, ya que toda la información que se obtiene será manejada como oficial y manteniendo la confidencialidad del informante (Secretaría de Salud 1987), y Código de Bioética que se enmarca en la Declaración Mundial de Helsinki (Asociación Médica Mundial, enmendada en la 52a Asamblea Mundial de Edimburgo en 2000; cumpliendo con las recomendaciones (Revisada en Tokio 1975).
} 
REDES- Revista hispana para el análisis de redes sociales

Vol.17,\#8, Diciembre 2009

http: //revista-redes.rediris.es

atributos de cada alter, tipos de relaciones y su contenido, e identifica los elementos poco vinculados y la conectividad externa, así como el registro de recursos institucionales. También detecta las relaciones específicas que pueden ser comparadas con la red que se manifiesta en otro tipo de situación; cambios y diferencias en densidad, extensión, función, etcétera.

\section{Análisis de resultados}

En ambos grupos se ha aplicado el cuestionario de Fernández-Ballesteros y Zamarrón ${ }^{8}$ que mide la calidad de vida, para ser utilizado en la evaluación previa y posterior a las intervenciones, como un referente general del impacto de las actividades que ya se han iniciado (las investigaciones concluyen en 2008); por lo que la primera tabla corresponde a la percepción del enfermo sobre la calidad de vida que tiene, y la de los cuidadores, influidos por la responsabilidad asumida (ver tabla 1 ).

En donde se puede observar como los egos de las redes perciben su condición de vida en las 9 áreas que comprende el cuestionario, siendo la de integración social una de las más afectada en los dos grupos 2.72 y 1.71 respectivamente (puntaje de 1-4), contrastando con la que se refiere a satisfacción con la vida en 3.00 y 3.03 (puntaje de 1-4), aun en su condición de vejez y cargas del cuidador; situación similar con cuidadores del estudio de 2002. Se observa que a medida que los enfermos se hacen más dependientes, recae una mayor responsabilidad sobre el cuidador principal, quien a su vez está expuesto a un mayor trabajo, limitando el tiempo disponible para establecer o mantener sus relaciones sociales.

Se manifiesta un progresivo incremento de hombres cuidadores (30\%) ya que era un ámbito feminizado; muy probablemente por las condiciones de transición demográfica que están generando nuevos escenarios sociales en el envejecimiento: baja tasas de nacimiento, elevación en la esperanza de vida, consolidación de otras formas de convivencia familiar; expectativas económicas complejas, continuos flujos de migración, incremento en la participación de la mujer como fuerza laboral, que concurren al debilitamiento en lazos de parentesco y disminución en las posibilidades de cuidados en familia.

\footnotetext{
${ }^{8}$ Fernández Ballesteros y Zamarrón, CUBRECAVI (Cuestionario Breve de calidad de vida 1996), en "Calidad de Vida en la vejez en los distintos contextos", Pirámide, Madrid, España.
} 
REDES- Revista hispana para el análisis de redes sociales

Vol.17,\#8, Diciembre 2009

http://revista-redes.rediris.es

\begin{tabular}{|c|c|c|c|}
\hline & ÁREA & $\begin{array}{c}\text { GPG } \\
\text { Promedio }\end{array}$ & $\begin{array}{c}\text { GC } \\
\text { Promedio }\end{array}$ \\
\hline 1 & Salud & 1.68 & 3.04 \\
\hline 2 & I ntegración social & 2.72 & 1.71 \\
\hline 3 & Habilidades funcionales & 1.69 & 3.55 \\
\hline 4 & Actividad y ocio & 1.82 & 2.21 \\
\hline 5 & Calidad ambiental & 2.92 & 2.52 \\
\hline 6 & Satisfacción con la vida & 3.00 & 3.03 \\
\hline 7 & Educación & 1.70 & 2.89 \\
\hline 8 & Ingresos & 2.00 & 1.69 \\
\hline 9 & Servicios & 2.00 & 2.01 \\
\hline
\end{tabular}

Tabla 1. Percepción de calidad de vida (cubrecavi).

En los dos grupos estudiados, la red personal esta integrada principalmente por familiares directos, destacando el apoyo de hijas e hijos, hermanas y hermanos del entrevistado con una media de edad del 43.5 y 51.9 años, respectivamente; en su mayoría casados. En el primer grupo el $64 \%$ se trata de familias extensas y en el segundo un 58.3\% son nucleares. En este último caso, más de la mitad corresponde a familias compuestas únicamente por el enfermo y su cuidador.

Cuentan en promedio con 7.60 y 3.95 de elementos en su red personal respectivamente, lo que marca una gran diferencia; probablemente por tratarse en el primero, de lazos del enfermo (red directa de enfermo); en el segundo, la red esta determinada por su actual condición de cuidador principal (red indirecta del enfermo).

Se identifican 24 y 32 diferentes categorías de vínculos, con más alta frecuencia en familiares que constituyen los lazos fuertes y los débiles como apoyos complementarios, integrado por familiares de segundo y tercer grado, así como por personas sin parentesco (ver tabla 2). En cuanto a los apoyos que proporcionan, se ubica con más alta frecuencia a los de tipo afectivo en los dos grupos con 33.60\% y 31.49\%; el económico es el que menos proporcionan, $15.60 \%$ y $22.55 \%$ respectivamente (ver tabla 3 ).

La gran mayoría de los entrevistados del primer grupo no asisten a grupos de ayuda del Hospital, pero el $10 \%$ va a grupos del INAPAM (actividades manuales y recreativas). Los acompañantes de pacientes hospitalizados, en todos los casos, se apoyan y ayudan entre quienes comparten la sala. 
REDES- Revista hispana para el análisis de redes sociales

Vol.17,\#8, Diciembre 2009

http://revista-redes.rediris.es

Los cuidadores asisten en un 33.3\% al grupo de la Residencia de día, refieren que carecen de alguien que cuide al enfermo en el horario que estas reuniones se efectúan (una tarde al mes), también un $8.3 \%$ concurre a otros grupos.

Con relación al acceso a servicios de salud, la totalidad de la población del primer grupo es derechohabiente del ISSSTE, y todos a excepción de una persona, tienen acceso a otro tipo de servicios médicos, lo que refiere una gran movilidad y uso indiscriminado de estos de recursos.

\begin{tabular}{|c|c|c|c|c|c|}
\hline \multicolumn{6}{|c|}{ Con vínculos de parentesco } \\
\hline \multicolumn{3}{|c|}{ Mujeres } & \multicolumn{3}{|c|}{ Hombres } \\
\hline CATEGORÍ A & GPG & GC & & GPG & GC \\
\hline Madre & 0.0 & 2.2 & Padre & 0.0 & 2.2 \\
\hline Hija & 24.1 & 16.8 & Hijo & 15.7 & 16.8 \\
\hline Nieta & 12.3 & 4.3 & Nieto & 9.2 & 3.2 \\
\hline Sobrina & 5.2 & 0.0 & Sobrino & 3.7 & 3.2 \\
\hline Hermana & 3.4 & 14.7 & Hermano & 2.6 & 10.5 \\
\hline Tía & 0.0 & 2.2 & Tío & 0.0 & 2.2 \\
\hline Nuera & 2.9 & 1.1 & Yerno & 5.0 & 1.1 \\
\hline Esposa & 0.8 & 3.2 & Esposo & 1.0 & 5.3 \\
\hline Cuñada & 1.0 & 1.1 & Cuñado & 1.8 & 0.0 \\
\hline Prima & 0.5 & 2.2 & Primo & 0.3 & 1.1 \\
\hline Bisnieta & 0.5 & 0.0 & Bisnieto & 0.8 & 0.0 \\
\hline \multicolumn{6}{|c|}{ Sin vínculos de parentesco } \\
\hline Amiga & 5.5 & 1.1 & Amigo & 0.8 & 1.1 \\
\hline Vecina & 1.8 & 1.1 & Vecino & 0.8 & 0.0 \\
\hline Ahijada & 0.0 & 0.0 & Ahijado & 0.3 & 0.0 \\
\hline Cuidadora \$ & 0.0 & 2.2 & Cuidador \$ & 2.1 & 0.0 \\
\hline Enfermera \$ & 0.0 & 0.0 & Enfermero \$ & 0.0 & 1.1 \\
\hline Total & $58 \%$ & $52.2 \%$ & & $42 \%$ & $47.8 \%$ \\
\hline \multicolumn{6}{|c|}{$\begin{array}{l}\text { Fuentes: } n=50 \text { Grupo Enfermos Geriátricos (2005-2007) y } \\
n=60 \text { Grupo de Cuidadores (2006-2007) } \\
\text { \$ Cuidadora-cuidador y enfermera-enfermero pagados. }\end{array}$} \\
\hline
\end{tabular}

Tabla 2. Elementos de la red personal (alteri)

En el grupo de cuidadores el 95\% de los enfermos tiene acceso al IMSS o al ISSSTE $^{9}$ y un $11.7 \%$ a ambos; en tanto que el cuidador tiene un $78.3 \%$ de acceso a diversos servicios médico, de ellos, el $70 \%$ es derechohabiente del IMSS o del ISSSTE, y una persona cuenta con ambos. El 6.7\% utiliza además servicios privados. Solo una persona indica no contar con ningún servicio médico y el $16 \%$ no contesto.

Con estos datos se pueden sacar algunas conclusiones, pero sobre todo, permiten iniciar acciones en cada grupo; especialmente con los cuidadores y dentro del marco del Megaproyecto, para: trabajo con su red personal, poner a su disposición información actualizada y confiable; educación y entrenamiento referente a demencias y el Alzheimer, efectos en el paciente y en el propio cuidador; servicios

\footnotetext{
${ }^{9}$ El Instituto Mexicano del Seguro Social (IMSS) y el Instituto de Seguridad y Servicios Sociales para los Trabajadores estado del (ISSSTE), son los dos principales servicios de seguridad social en México.
} 
REDES- Revista hispana para el análisis de redes sociales

Vol.17,\#8, Diciembre 2009

http: // revista-redes.rediris.es

psicológicos y sociales de manera continua, orientados a mejorar su calidad de vida. Fomento hábitos de vida saludables; grupos de ayuda y autoayuda, la sensibilización más allá de sus grupos y la Institución.

\begin{tabular}{|l|l|l|}
\hline Tipo & \multicolumn{1}{|c|}{ GPG } & \multicolumn{1}{c|}{ GC } \\
\hline Afecto/confianza & 33.60 & 31.49 \\
\hline Cuidados/compañía & 27.60 & 27.66 \\
\hline Información/servicios & 23.20 & 18.30 \\
\hline Económico/material & 15.60 & 22.55 \\
\hline Total & $100 \%$ & $100 \%$ \\
\hline $\begin{array}{l}\text { Fuente: } n=50 \text { Grupo Enfermos Geriátricos (2005-2007) y } \\
n=60 \text { Grupo de Cuidadores (2006-2007) }\end{array}$ \\
\hline
\end{tabular}

Tabla 3. Apoyo otorgado por la red personal.

\section{Conclusiones}

1. El capital social en el ámbito de la salud ejerce una función protectora que contribuyen al bienestar y la calidad de vida; asociado al conocimiento de la vejez representa la oportunidad para analizar la correlación que existe entre esta etapa de vida y la red social; ya que a medida que se envejece, es común que disminuya la actividad social del individuo y con ello, las formas de mantener los contactos con los demás sujetos que integran su red personal, sobre todo cuando presenta algún padecimiento crónico degenerativo, o algún tipo de demencia, en cuyo caso, pasan a depender un cuidador principal.

2. Es fundamental el estudio y recuperación de estas redes relacionales, porque representan lazos que añaden valor a los individuos y que en algunas culturas se manifiesta como un stock de cooperación con que cuenta una sociedad; llegando a constituir un elemento de sobrevivencia en grupos vulnerables y la base de la solidaridad, alcanzado gran significado ante procesos de enfermedad, pérdida o crisis.

3. En este trabajo se comparan algunos de los resultados iniciales en dos estudios de redes personales, con objeto de promover mejor y mayor participación de los integrantes, en beneficio del enfermo, su cuidador y la familia. En ambos casos la distribución de datos demográficos es similar a otros estudios realizados con enfermos de padecimientos crónico-degenerativos y los cuidadores principales, pero marca diferencias sustanciales en los datos de socialización entre los grupos estudiados, sobre todo en el número de elementos en red. Las hermanas y los hermanos, hijas e hijos son los que dan el mayor apoyo al enfermo y a los cuidadores principales. En todos los casos la red personal del cuidador beneficia directa o indirectamente al enfermo. 
REDES- Revista hispana para el análisis de redes sociales

Vol.17,\#8, Diciembre 2009

http: // revista-redes.rediris.es

4. En ambos grupo estudiados se requiere reforzar y ampliar esa red, buscando disminuir el desgaste y mejorar la calidad de vida de los entrevistados, a partir de una mayor participación con la familia de origen y su ampliación a través de mayor integración con amigos, vecinos, los grupos de pertenencia, comunitarios, religión, o trabajo; así como la formalización de vínculos con las instituciones públicas o privadas que guardan relación con las necesidades específicas de los grupos estudiados.

5. En el grupo de cuidadores se cuentan con una escasa, pero importante red personal y social, de la cual reciben apoyos de tipo emocional, social, instrumenta y económico. Requiere de estructuras estables, que permita a los miembros del afrontamiento de sus necesidades y sentimientos, derivados de las características de esta enfermedad. También es importante que los cuidadores acudan al grupo de ayuda y autoayuda.

6. Finalmente, hacer mejor cada día las acciones de salud, demanda mantener actualizada la formación profesional e incorporar distintas tendencias, como pude ser la teoría, análisis y representación de redes sociales (TARS).

\section{Agradecimientos}

Nuestro agradecimiento a las instituciones por las facilidades otorgadas para el desarrollo de esta investigación, particularmente a los enfermos y los cuidadores, por su confianza y entusiasta participación.

\section{Bibliografía}

Bellón JA, Delgado A, Luna del CD y Lardelli CP. (1996). Validez y fiabilidad del cuestionario de apoyo social funcional Duke-UNC-11. Aten Primaria, Vol. 28, pp.153-163.

Caraveo AJ . (1997) "Epidemiología de los trastornos depresivos". Rev Psiquiatría, Vol. 13, pp. 1-4.

De Federico A. (2005). “El análisis dinámico de redes sociales con SIENA, método, discusión y aplicación". Empiria. Revista de Metodología de Ciencias Sociales. No. 10, Julio-Diciembre, 2005, pp.151-181. ISSN: 1139-5737.

De la Revilla AL, Ballón E, Dios LJ, Delgado A, Prado MA y Fleitas L. (1991). Validación de una escala de apoyo social funcional para su uso en la consulta del médico familiar. Atención Primaria, Vol. 8 pp. 688-691.

De Grande PE. (2007). Redes sociales y espacio urbano. V Mesa Hispana para el análisis de redes sociales.XXVII International Sunbelt Social Network Conference. Corfú, Grecia, 1 de mayo de 2007.

Díaz Veiga T. (1987). Evaluación del apoyo social. En R. Fernández-Ballesteros: El ambiente, análisis psicológico. Pirámide, Madrid, España. 
REDES- Revista hispana para el análisis de redes sociales

Vol.17,\#8, Diciembre 2009

http: //revista-redes.rediris.es

Fernández-Ballesteros R, Zamarrón MD y Maciá C. (1996). Calidad de Vida en la vejez en los distintos contextos. Pirámide, Madrid, España.

Infante CC. (1990). “Utilización de servicios de atención prenatal: influencia de la morbilidad percibida y de las redes sociales de ayuda". Salud Pública de México, Vol. 32, pp. 419-429.

Lin, N. (2001). Social Capital: A Theory of Social Structure and Action. Cambridge: Cambridge University Press.

Granovetter M. (1973). "The Strength of weak Ties". American Journal of Sociology, 78(6), 1360-1380.

González MA, Terán M, Ponce ER y Sánchez LE (2003). “Salud del cuidador y sus redes e apoyo en una clínica de medicina familiar de la Ciudad de México". ArchMedFam; 5(2):47-52. http://www. geocites.com/archmedfam. pdf

Loucks EB, Berkman LF, Gruenewal TL and Seeman TE (2006). "Relation of Social Integration to Inflammatory Marker Concentratons in Men and Women 70 to 79 Years". Am J Cardiol (97): 1010-1016.

Litman TJ. (1982). "La familia, la atención y la salud: un estudio socioconductista". En: Jaco E. (comp.) pp. 123-173.

Maya I. (2002). "Tipos de redes personales de los inmigrantes y adaptación psicológica". Revista Redes, Vol.1, No.4, Enero. http://revista-redes.rediris.es

Molina JL. (2001). El análisis de redes sociales. Una introducción. Serie General Universitaria No. 10, Bellatierra, España.

Molina J L. (2005). Redes personales: un programa de investigación.

\section{En: www.revista-redes.rediris.es/webredes/}

Molina JL. (2005). “El estudio de las redes personales: contribuciones, métodos y perspectivas". Empiria, Julio-Diciembre 10 (71-106)

\section{http://seneca.uab.es/antropologia/Egoredes/public_archivos/redes_personales .pdf}

Requena SF. (1996). Redes sociales y cuestionarios. Cuadernos metodológicos No. 18. ClS, España.

Reyes S, Bobadilla JL, Karchmer S y Martínez L. (1998). "Efectos de la muerte materna sobre la dinámica familiar y la sobrevivencia infantil". Ginecología y obstetricia de México, Vol. 66, México, pp. 428-433.

Rodríguez M y Guerrero F. (1997). “Importancia del apoyo familiar en el control de la glucemia". Salud Pública de México, Vol. 39, pp. 44-47.

Rodríguez Y, Díaz OC y García CC. (1995). “La trascendencia de los grupos de apoyo en el manejo integral de los enfermos crónicos". Gaceta Médica Mexicana, Vol 131, pp. 329-334.

Terán M. (2001). Las redes sociales en atención a la salud. En: La política social en la transición. ENTS-UNAM-Plaza y Valdez, México. Pp.468-484. 